\title{
Obtención de celulosa cristalina de hoja de maíz de Metztitlán Hidalgo
}

\author{
Obtention of crystalline cellulose from corn leaves of Metztitaln Hidalgo

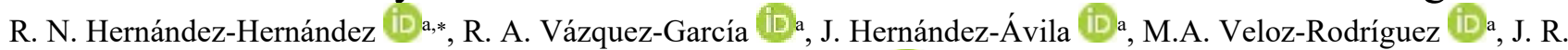 \\ Villagómez-Ibarra $\mathbb{B D}_{\mathrm{b}}$ \\ a Área Académica de Ciencias de la Tierra y Materiales, Universidad Autónoma del Estado de Hidalgo, 42184, Pachuca, Hidalgo, México. \\ ${ }^{b}$ Área Académica de Química, Universidad Autónoma del Estado de Hidalgo, 42184, Pachuca, Hidalgo, México.
}

\section{Resumen}

La necesidad del aprovechamiento óptimo de los recursos ha incrementado el interés de estudiar otras fuentes de fibras celulósicas para emplear en una gran variedad de aplicaciones. Este artículo se enfoca en la extracción de celulosa de hoja de maíz (Zea mays) mediante dos procedimientos experimentales: ácido y etanosolv. La caracterización se realizó por espectroscopia infrarroja (FT-IR) y difracción de rayos X (DRX). Los espectros FT-IR muestran las vibraciones características presentes en los materiales lignocelulósicos y los difractogramas señalan los ángulos de difracción distintivos de celulosa I.

Palabras Clave:

Celulosa, Hoja de maíz, Etanosolv, Caracterización, Zea mays

\begin{abstract}
The need for optimal resource utilization has been increased the interest in studying other sources of cellulosic fibers for use in a wide variety of applications. This article focuses on the extraction of cellulose from corn (Zea mays) leaves using two experimental procedures: acid and ethanosolv. Characterization was made by infrared spectroscopy (FT-IR) and X-ray diffraction (XRD). The FTIR spectra shows the characteristic vibrations present in the lignocellulosic materials and XRD diffractogram points out the distinctive diffraction angles of cellulose I.
\end{abstract}

Keywords:

Cellulose, Corn husk, Organosolv, Characterization, Zea mays.

\section{Introducción}

Actualmente, en todo el mundo se vive el reto de satisfacer las necesidades de la población, destacando la alimentación y el cuidado al medio ambiente, así como el óptimo aprovechamiento de los recursos. México en especial, cuenta con las condiciones edafoclimáticas que le permiten cultivar 264 especies vegetales (Sosa \& Ruíz, 2017) destacando entre ellas el maíz y el frijol debido a su producción, valor económico y cultural (Pérez, 2020); la superficie sembrada con maíz en el año 2019 fue de 6.6 millones de hectáreas (INEGI, 2019), ocupando el octavo lugar en producción mundial para el periodo 2020/21 (United States Department of Agriculture, 2021)

Como en otras regiones de México, los productores se benefician económicamente de la venta del elote, del grano y adicionalmente del totomoxtle (Sierra et al., 2016); este último se refiere a las hojas que protegen la mazorca hasta su maduración, cuyas propiedades como tamaño, espesor, y adecuadas condiciones mecánicas y sanitarias, las convierten en hojas útiles para elaborar tamales (Alvarado et al., 2020; Lascurain et al.,
2017), que son uno de los platillos más representativos y famosos de México, así mismo, son utilizadas para elaborar manualidades como muñecas y flores (King, 2007)

Por otra parte, el uso integral de la cosecha de maíz va desde la venta del grano para consumo humano y animal (Godina et al., 2020) y de la hoja de maíz apta, hasta el uso de los residuos para abono de la tierra o como forraje para el ganado (Velázquez et al., 2002), no obstante, siendo una práctica efectiva de eliminación de residuos es usual que se lleve a cabo la quema de estos (Erenstein, 1999).

En Metztitlán, Hidalgo, la agricultura es la actividad económica de mayor importancia, el $73 \%$ de la población se dedica a ella y se siembran maíz, frijol, r, jitomate, entre otros (López et al., 2015). En las comunidades pertenecientes a esta región agrícola, los habitantes dedicados a la venta de maíz, refieren que el porcentaje de hoja que no cumple con las características para la venta y comercialización, se considera como residuo y se quema; esta actividad a largo plazo podría derivar en pérdidas por erosión del suelo, degradándolo constantemente (Nele \& Bram, 2015).

\footnotetext{
*Autor para la correspondencia: lazcarr_222@hotmail.com

Correo electrónico: lazcarr_222@hotmail.com (Régula Nallely Hernández-Hernández), rosavg@uaeh.edu.mx (Rosa Ángeles Vázquez-García), herjuan@uaeh.edu.mx (Juan Hernández-Ávila), Maveloz70@yahoo.com.mx (María Aurora Veloz-Rodríguez) y jrvi@uaeh.edu.mx (José Roberto Villagomez-Ibarra) 
Los residuos agrícolas, la madera, las plantas acuáticas, pastos, entre otros cuerpos vegetales, contienen celulosa, que es el biopolímero más abundante de la tierra; su estructura está formada por enlaces 1,4- $\beta$ glucosídicos y el grado de cristalinidad depende de la fuente de la que es aislada; además, por la presencia de tres grupos $\mathrm{OH}$ en su unidad estructural, puede reaccionar con alcoholes, esteres o éteres (Gañán et al., 2017); todo esto le confiere propiedades como biocompatibilidad, biodegradabilidad, estabilidad térmica y química, renovabilidad, bajo costo y sin toxicidad, que la hacen altamente atractiva para aplicaciones como la fabricación de papel, textiles, películas, rellenos, materiales para construcción, entre otros, Figura 1, (Qi, 2017).

Autores como Huang et al., 2017, aislaron nanocristales de celulosa de tallos de maíz utilizando la hidrósilis con ácido sulfúrico, en donde la cristalinidad pasó de $33.2 \%$ a $69.2 \%$; así mismo Singh et al., 2019, estudiaron el aislamiento de celulosa microcristalina de rastrojo de maíz por el método de hidrólisis ácida variando la concentración de los reactivos químicos y agregando dos etapas más al proceso: tratamiento alcalino y blanqueado, esto les permitió obtener un indice de cristalinidad de 91.26\%.

Por otra parte, el tratamiento por organosolv es un método modificado de pulpeo empleado para la generación de bioetanol, sin embargo, permite obtener la fracción de celulosa en forma sólida al remover las fracciones de lignina y hemicelulosa en forma líquida a través del empleo de solventes orgánicos como etanol, metanol, ácido acético, entre otros, con o sin un catalizador ácido como el ácido sulfurico (Nazli \& Karaosmanoglu, 2018); el estudio realizado por Zhang \& Liu, 2018, indica que despues de la solubilización de madera de Eucalyptus con organosolv ocurrió un incremento en la cristalinidad (69.1\%), así mismo Hernández et al., 2018, reportan que después del tratamiento el grado de cristalinidad del Agave tequilana fue de $66 \pm 2.24 \%$.

Por lo anterior, es de interés desarrollar métodos que permitan el empleo de los residuos de hoja de maíz como materia prima para la obtención de celulosa de fuentes no maderables y que, al mismo tiempo, permitan el uso integral de los recursos en esta región del estado de Hidalgo.

\section{Metodología}

\subsection{Zona de estudio y recolección de muestras}

Las hojas de maíz fueron donadas por una familia de la comunidad del Pedregal de Zaragoza en Metztitlán $\left(20^{\circ} 35^{\prime} 04^{\prime \prime} \mathrm{N}\right.$ $\left.98^{\circ} 45^{\prime} 47^{\prime \prime} \mathrm{O}\right)$, Hidalgo, correspondientes a la primera temporada de siembra de marzo a junio del 2020 .

\subsection{Preparación de la muestra para la caracterización química}

Las hojas secas fueron trituradas en una licuadora marca Oster, hasta obtener fibras de $2 \mathrm{~cm}$ de largo. De acuerdo a la norma T-264 cm-97, la muestra debe encontrarse libre de extraíbles, por lo tanto, se llevó a un proceso extractivo Soxhlet con una mezcla de solventes, etanol:tolueno (1:2 por volumen) por 6 horas, seguido de un enjuague con etanol y posteriormente una segunda extracción con etanol al 95\% durante 4 horas. Al finalizar, la muestra se lavó con $500 \mathrm{~mL}$ de agua destilada caliente y se secó a temperatura ambiente.

\subsection{Caracterización química}

\subsubsection{Determinación de holocelulosa (método Jayme-Wise)}

Se pesaron $5 \mathrm{~g}$ de la muestra libre de extraíbles, se colocaron en un matraz Erlenmeyer de $500 \mathrm{~mL}$, se agregaron $1.5 \mathrm{~g}$ de clorito de sodio $\left(\mathrm{NaClO}_{2}\right)$ a $160 \mathrm{~mL}$ de agua, más 10 gotas de ácido acético concentrado y se colocó en baño María a $80^{\circ} \mathrm{C}$ durante 1 hora. Posteriormente, se agregaron nuevamente $1.5 \mathrm{~g}$ de clorito de sodio $\left(\mathrm{NaClO}_{2}\right)$ y 10 gotas de ácido acético, se agitó y se dejó en reposo hasta completar 2 horas, después se lavó y filtró con 500 $\mathrm{mL}$ de agua bidestilada caliente y finalmente con $250 \mathrm{~mL}$ de acetona. La muestra se dejó secar en horno durante 12 horas a $70^{\circ} \mathrm{C}$ hasta peso constante, se dejó enfriar en desecador y se pesó determinando así el contenido de holocelulosa.

\subsubsection{Determinación de lignina por el método Klason}

De acuerdo a la norma T $222 \mathrm{~cm}-88$, en un vaso de precipitados se colocaron $0.5 \mathrm{~g}$ de muestra libre de extraíbles, se le agregaron $15 \mathrm{~mL}$ de $\mathrm{H}_{2} \mathrm{SO}_{4}$ y se mantuvo en agitación 2 horas, al finalizar se vació a un vaso de $1 \mathrm{~L}$ y se realizó una disolución al $4 \%$ de $\mathrm{H}_{2} \mathrm{SO}_{4}$ agregando $560 \mathrm{~mL}$ de agua destilada, se dejó en ebullición suave por 4 horas, añadiendo agua caliente para mantener el volumen constante, posteriormente se dejó enfriar a temperatura ambiente. Cuando la muestra decantó, se filtró y se llevó al horno a $50{ }^{\circ} \mathrm{C}$ durante 24 h, se pesó, determinando así el contenido de lignina.

\subsubsection{Determinación de celulosa por el método Kurschner y Hoffer.}

Se pesó $1 \mathrm{~g}$ de materia libre de extraíbles, se colocó en un matraz y se añadieron $20 \mathrm{~mL}$ de etanol y $5 \mathrm{~mL}$ de ácido nítrico. la mezcla se colocó en reflujo a baño maría por 30 minutos, se filtró y el sólido se sometió a una segunda digestión de 30 minutos con $25 \mathrm{~mL}$ de etanol-ácido nítrico, posteriormente, se filtró y la muestra sólida se llevó a una tercera digestión con $100 \mathrm{mLde}$ agua destilada por 1 hora, después se filtró y se lavó con agua destilada caliente, seguida de $100 \mathrm{~mL}$ de una solución saturada de acetato de sodio anhidro y por último con $500 \mathrm{~mL}$ de agua destilada caliente. Finalmente, el residuo se secó en horno a $100{ }^{\circ} \mathrm{C}$, se mantuvo en desecador y se pesó. El contenido de celulosa se determinó mediante la siguiente relación.

$$
\% \text { celulosa }=A * 100 W
$$

$\mathrm{A}=$ peso de la muestra seca después del tratamiento

$\mathrm{W}=$ peso seco de la muestra libre de extraíbles

\subsection{Hidrólisis ácida (CMAc)}

En un matraz bola se colocaron $15 \mathrm{~g}$ de hoja de maíz libre de extraíble y se les agregó una mezcla de $200 \mathrm{~mL}$ de etanol y $50 \mathrm{~mL}$ de ácido sulfúrico, se llevó a reflujo con agitación en baño María a $85{ }^{\circ} \mathrm{C}$ por 30 minutos, posteriormente se filtró el líquido y se reservó, a la muestra sólida se le agregaron otros $250 \mathrm{~mL}$ de la mezcla etanol-ácido sulfúrico y se repitió el proceso, posteriormente la muestra sólida se llevó a reflujo por 1 hora con $300 \mathrm{~mL}$ de agua bidestilada con agitación a $100{ }^{\circ} \mathrm{C}$. La muestra fue decantada y lavada hasta $\mathrm{pH}$ neutro. Finalmente se mantuvo en un horno a $75^{\circ} \mathrm{C}$ por 24 horas. 


\subsection{Etanosolv (CMSo)}

Se pesaron $10 \mathrm{~g}$ de muestra y se colocaron en un matraz bola con $100 \mathrm{~mL}$ de agua, $80 \mathrm{~mL}$ de etanol y $2 \mathrm{~mL}$ de ácido clorhídrico como catalizador; y se colocó en baño María con reflujo y agitación a $85^{\circ} \mathrm{C}$ por 1 hora, transcurrido el tiempo, se filtró y el líquido se reservó. Posteriormente, a la muestra se le agregaron $250 \mathrm{~mL}$ de agua destilada, repitiendo el proceso a $100^{\circ} \mathrm{C}$. Una vez filtrada, la muestra sólida se enjuagó con agua destilada caliente, después con una solución de bicarbonato de sodio y finalmente con agua destilada caliente hasta $\mathrm{pH}$ neutro, el sólido se secó en horno por 24 horas a $75^{\circ} \mathrm{C}$.

\subsection{Reducción de tamaño}

Se pesaron $1.5 \mathrm{~g}$ de las muestras CMAc y CMSo y se colocaron en contenedores de acero con 3 bolas de polietileno de ultra alto peso molecular, se molieron por 3 horas en lapsos de 15 minutos, utilizando un molino de bolas de alta energía Spex 8000D de 2000 rpm. Al finalizar, el polvo obtenido fue tamizado con una malla $80 \mu \mathrm{m}$.

\subsection{Caracterización de la celulosa}

\subsubsection{Difracción de rayos $x$}

Los cambios en la estructura cristalina de las muestras se analizaron mediante un difractómetro de Rayos X marca Bruker AXS modelo D2 Phaser, con radiación de $\mathrm{Cu} \mathrm{K} \alpha$ (1.5405). El índice de cristalinidad (CrI) se calculó con la ecuación 1 conforme a los picos característicos de la celulosa correspondientes a su fracción cristalina y amorfa.

$$
\operatorname{CrI}(\%)=\left(1-\frac{I_{A m}}{I_{002}}\right) * 100
$$

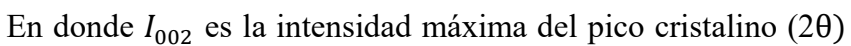
en $22^{\circ}$ e $I_{A m}$ es la intensidad del pico amorfo $(2 \theta)$ en $16^{\circ}$ para celulosa I (Rewini et al., 2018), (Torres et al., 2017) y (Bolio et al., 2011).

\subsubsection{Espectroscopia infrarroja}

Las muestras se analizaron en un espectrofotómetro FTIR Frontier marca Perkin Elmer.1

\section{Análisis y discusión de resultados}

\subsection{Caracterización química}

Se analizaron los porcentajes de holocelulosa, celulosa y lignina, de acuerdo a las Normas Tappi, comparando con el estudio realizado por (Prado et al., 2012) en donde se observa que los porcentajes correspondientes a la holocelulosa y lignina son similares, sin embargo el porcentaje de celulosa en este estudio es mayor; esta variación puede deberse a la diferencias climaticas y de origen de la materia prima (Parejo et al., 2019), (Yepes \& Silveira, 2011), convirtiendo a la hoja de maíz en una fuente adecuada para la extracción de este biopolímero.
Tabla 1. Composición química de la hoja de Zea mays, comparación con resultados publicados.

\begin{tabular}{cccc}
\hline Compuesto & Holocelulosa & Celulosa & Lignina \\
\hline Hoja de maíz & 83.9 & 56.16 & 21.05 \\
Hoja de maíz (Prado & 78.86 & 43.14 & 23 \\
et al., 2012) & & & \\
\hline
\end{tabular}

\subsection{Difracción de rayos $x$}

La Figura 1, compara los patrones de difracción de la celulosa microcristalina Avicel PH-101 (CC) con la materia prima y las celulosas CMAc y CMSo, revelando los picos característicos de la celulosa I localizados en aproximadamente $2 \theta=16^{\circ}, 22^{\circ}$ y $35^{\circ}$ correspondientes a los planos hkl 110, 200 y 004 respectivamente (Kampeerapuppun, 2015), (Hemmati et al., 2018), (Das et al., 2014) (Pokhrel et al., 2020), para CC (1.b) se presentan en $15.6^{\circ}$, $22.5^{\circ}$ y $34.6^{\circ}$, en la materia prima (1.a) se observa la región amorfa que abarca aproximadamente de $10.97^{\circ}$ a $14.78^{\circ}$ y una señal en $21.8^{\circ}$, después de los tratamientos para CMAc (1.d) se advierten picos en $16.2^{\circ}, 22.3^{\circ}$ y $34.8^{\circ}$; y en CMSo (1.c) se forman en $15.7^{\circ}, 21.6^{\circ}$ y $34.5^{\circ}$. Así mismo, se observa un pico en $2 \theta=18^{\circ}$, sin embargo, no fue asignado en esta etapa del estudio.

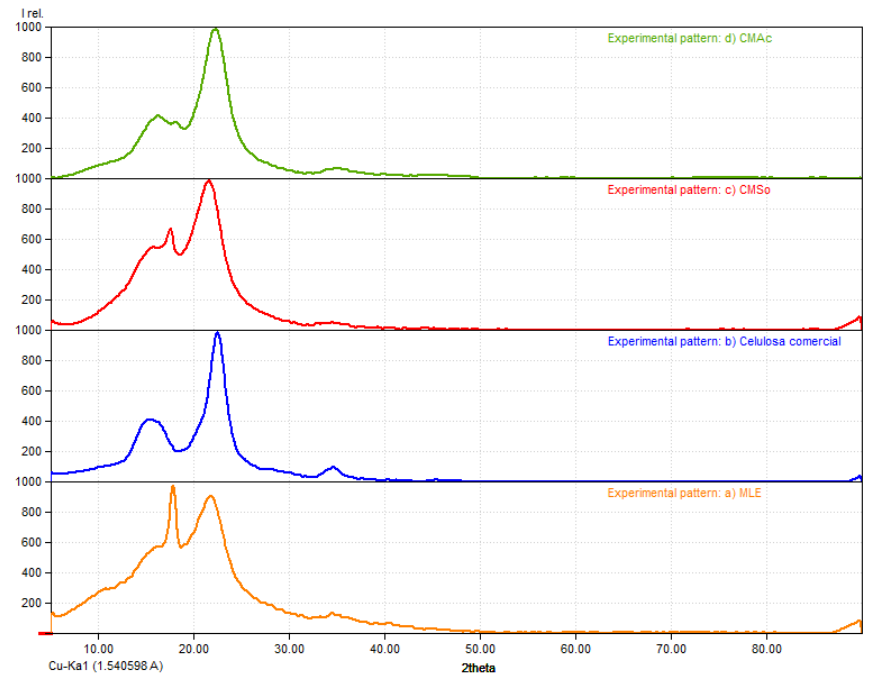

Figura 1. Difractogramas de la materia prima libre de extraíbles, y de la hoja de maíz tratada, comparados con celulosa microcristalina Avicel. 1.a) materia prima libre de extraíbles, 1.b) celulosa microcristalina Avicel, 1.c) celulosa etanosolv y 1.d) celulosa ácida.

Por otra parte, en el tratamiento con ácidos, la región amorfa de la celulosa se hidroliza resultando en un aumento de la cristalinidad, la hidrolisis está influenciada por las propiedades estructurales de la materia prima como el grado de cristalinidad, el área superficial, el grado de polimerización y la porosidad de la muestra; en los difractogramas (Figura 1) se puede observar que el índice de cristalinidad obtenido con (2) de la materia prima es de $36.5 \%$, mientras que el de la muestra CMSo (1.c) es de $45.2 \%$ y para CC (1.a) y CMAc (1.b) corresponden a $54.53 \%$ y $53.35 \%$ respectivamente (Sebe et al., 2012), por lo tanto, el tratamiento ácido remueve mejor la materia amorfa no celulósica en comparación con el método etanosolv, sin embargo ambos métodos promueven la formación de celulosa cristalina aunque en menor porcentaje que los reportados en los estudios previos antes mencionados. 


\subsection{Análisis espectroscópico}

El espectro de FT-IR se obtuvo en la región de 4000 a $600 \mathrm{~cm}^{-1}$; como se observa en la Figura 2, el espectro muestra los picos característicos de la celulosa, hemicelulosa y lignina de las muestras de materia prima (MP) (a), CMAc (b) y CMSo (c). La banda de absorción en $3338 \mathrm{~cm}^{-1}$ corresponde al estiramiento simétrico de los enlaces O-H (Dutta, 2017); el pico en $2892 \mathrm{~cm}^{-1}$ representa al estiramiento asimétrico de los enlaces $\mathrm{C}-\mathrm{H}$ de los alquilos y alifáticos presentes en celulosa, hemicelulosa y lignina (Velázquez et al., 2020). La banda presente en $1730 \mathrm{~cm}^{-1}$ indica los estiramientos de los enlaces $\mathrm{C}=\mathrm{O}$ de los grupos éster $\mathrm{y}$ carboxilo de la hemicelulosa, por ello se observa una mínima disminución en su intensidad después del tratamiento ácido (Kathirselvam et al., 2019), el pico presente en 1637 $\mathrm{cm}^{-1}$ corresponde a la vibración de flexión de los enlaces $\mathrm{O}-\mathrm{H}$ del agua absorbida por las fibras naturales (Yue et al., 2015). Las banda en $1430 \mathrm{~cm}^{-1}$ indica la vibración de los grupos $\mathrm{CH}_{2}$, es débil para la celulosa amorfa y en $1371 \mathrm{~cm}^{-1}$ se presenta el estiramiento alifático de la hemicelulosa (Singh et al., 2020) (Nagaraja Ganesh \& Muralikannan, 2016). El pico presente en $1236 \mathrm{~cm}^{-1}$ está asociado a los enlaces C-O del anillo de guayacil de la lignina y a sus vibraciones de estiramiento, esta señal es la disminución más notable entre los espectros, demostrando que la deslignificación parcial es más efectiva con el método ácido. Los picos en $1154 \mathrm{~cm}^{-1}$ y $1031 \mathrm{~cm}^{-1}$ pertenecen a las vibraciones de estiramiento asimétrico de los enlaces C-O-C de la celulosa (Kale et al., 2018) y (Singh et al., 2020). La banda de absorción en 893 $\mathrm{cm}^{-1}$ corresponde a la vibración de estiramiento de los enlaces C$\mathrm{O}-\mathrm{C}$ de los enlaces $\beta$-glucosídicos en la celulosa (Yue et al., 2015).

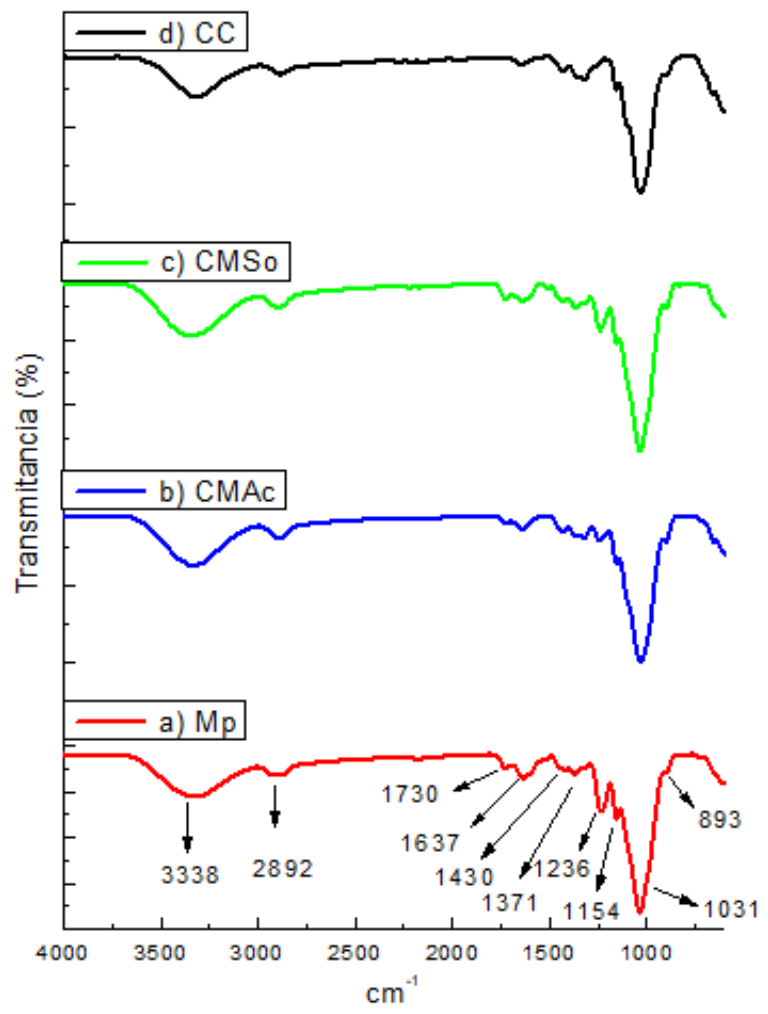

Figura 2. Espectros de FT-IR, a) materia prima, b) celulosa ácida, c) celulosa etanosolv y d) celulosa Avicel.

\section{Conclusiones}

Es posible obtener celulosa de la hoja de maíz de Metztitlán, cuya cristalinidad es similar a la de la celulosa microcristalina Avicel PH-101, sin embargo, es notable que tanto el método ácido como el etanosolv no remueven en su totalidad la lignina y hemicelulosa, por lo tanto, se considera conveniente someter a un tratamiento posterior de blanqueo para aumentar el grado de cristalinidad de la celulosa e incluso, variar las condiciones de procesamiento como tiempo, concentración del catalizador ácido, tipo de catalizador y solvente; complementar la caracterización con microscopia electrónica de barrido para observar la morfología de las fibras y determinar el rendimiento y la pureza de la celulosa. Finalmente es de vital importancia, asignar el pico presente en $2 \theta=18^{\circ}$ para determinar si se trata de algún contaminante que es eliminado con los tratamientos antes mencionados.

\section{Agradecimientos}

Se agradece al CONACYT por el apoyo otorgado mediante la beca 1034245 a R. N. Hernández Hernández, en el programa de Maestría en Ciencias de los Materiales que se realiza en el Área Académica de Ciencias de la Tierra y Materiales en el Instituto de Ciencias Básicas e Ingeniería de la Universidad Autónoma del Estado de Hidalgo.

\section{Referencias}

Alvarado Teyssier, R., Escobedo Garrido , S., Aceves Ruíz , E., Morales Jiménez, J., \& Mora Pérez , M. (2020). La cadena de valor de la hoja de maíz (totomoxtle) en el Valle de Puebla, México. Agricultura, sociedad y desarrollo, 17(4), 583-602. Recuperado de https://dialnet.unirioja.es/servlet/articulo? $\operatorname{codigo}=78$ 78872

Bolio López, G. I., Valadez González, A., Veleva, L., \& Andreeva , A. (2011). Whiskers de celulosa a partir de residuos agroindustriales de banano: obtención y caracterización. Revista Mexicana de Ingeniería Química, 10(2), 291-299. Recuperado de http://www.scielo.org.mx/scielo.php?script=sci_artte xt\&pid=S1665-27382011000200013

Das, A. M., Ali, A. A., \& Hazarika, M. P. (2014). Synthesis and characterization of cellulose acetate from rice husk: Eco-friendly condition. Carbohydrate Polymers, 342-349. doi:10.1016/j.carbpol.2014.06.00

Dutta, A. (2017). Fourier Transform Infrared Spectroscopy. En Spectroscopic Methods for Nanomaterials Characterization (págs. 73-93). Elsevier. doi:10.1016/B978-0-323-46140-5.00004-2

Erenstein, O. (1999). La conservacion de residuos de los sistemas de producción de maíz en Ciudad Guzmán y San Gabriel, Jalisco. CIMMYT. Recuperado de https://repository.cimmyt.org/bitstream/handle/1088 3/542/68089.pdf? sequence $=1 \&$ is Allowed $=y$ 
Gañán , P., Zuluaga , R., Castro , C., Restrepo Osorio, A., Velásquez Cock, J., Montoya , Ú., Vélez, L., Álvarez, C., Correa, C., Molina , C. (2017). Celulosa: un polímero de siempre con mucho futuro. Revista Colombiana de Materiales, 01-04. Recuperado de https://revistas.udea.edu.co/index.php/materiales/arti cle/view/328779/20785474

Godina Rodríguez, J. E., Garay Martínez, J. R., Mendoza Pedroza, S. I., Cancino, S. J., Rocandio Rodríguez, M., \& Lucio Ruíz , F. (2020). Rendimiento de forraje y composición morfológica de maíces nativos en condiciones semiáridas. Revista Mexicana de Ciencias Agrícolas(24), 59-68. doi:https://doi.org/10.29312/remexca.v0i24.2358

Hemmati, F., Mahdi Jafari, S., Kashninejad, M., \& Barani Motlagh, M. (2018). Synthesis and characterization of cellulose nanocrystals derived from walnut shell agricultural residues. International Journal of Biological Macromolecules. doi:10.1016/j.ijbiomac.2018.09.012

Hernández , J., Romero, V., Escalante , A., Toríz , G., Rojas , O. J., \& Sulbarán, B. (2018). Agave tequilana Bagasse as source of cellulose nanocrystals via organosolv treatment. Bioresourses, 3063-3614. Recuperado de https://ojs.cnr.ncsu.edu/index.php/BioRes/article/vie w/BioRes_13_2_3603_Hernandez_Agave_tequilana Bagasse_Cellulose

Huang, S., Zhou, L., Chun Li, M., Wu, Q., \& Zhou, D. (2017). Cellulose nanocrystals (CNCs) from corn stalk: Activation energy analysis. Materials, 1-13. doi:10.3390/ma10010080

INEGI. (2019). Encuesta nacional agropecuaria. Obtenido de https://www.inegi.org.mx/temas/agricultura/

Kale, R. D., Getachew Alemayeuh, T., \& Gorade, V. (2018). Extraction and characterization of Lignocelullosic fibres from Girardinia Bullosa (Steudel) Wedd. (Ethiopian Kusha Plant). Journal of Natural Fibers, 2-15. doi:10.1080/15440478.2018.1539940

Kampeerapuppun, P. (2015). Extraction and characterization of celullose nanocrystals produced by acid hydrolysis from corn husk. Journal of metals, materials and minerals, 25(1), 19-26. doi:10.14456/jmmm.2015.3

Kathirselvam, M., Kumaravel , A., Arthanasieswaran , V., \& Saravanakumar, S. (2019). Characterization of cellulose fibers in Thespesia populnea barks: Influence of alkali treatment. Carbohydrate Polymers, 178-189. doi:10.1016/j.carbpol.2019.04.063

King , A. (2007). Trade and Totomoxtle: Livelihood strategies in the Totonacan region of Veracruz, Mexico. Agriculture and Human Values, 24, 29-40. doi:DOI 10.1007/s10460-006-9031-3

Lascurain , M., López Binnquist , C., Avendaño , S., \& Covarrubias, M. (2017). The plants leaves used to wrap tamales in the Mexican State of Veracruz. Economic Botany, 71(4), 374-379. doi:doi:10.1007/s12231-017-9396-9
López Herrera, M., Romero Bautista , L., Ayala Sánchez , N., Soria Mercado , I. E., \& Portillo López , A. (2015). Problemática de contaminación en la zona agrícola de la Reserva de la Biosfera Barranca de Metztitlán, Hidalgo, México. Estudios en Biodiversidad, 1, 142150. Recuperado de https://digitalcommons.unl.edu/cgi/viewcontent.cgi? article $=1011 \&$ context $=$ biodiversidad

Nagaraja Ganesh, B., \& Muralikannan, R. (2016). Extraction and Characterization of Ligno-Cellulosic Fibers From Luffa Cylindrica Fruit. International Journal of Polymer Analysis and Characterization, 1-20. doi:10.1080/1023666X.2016.1146849

Nazli Borand, M., \& Karaosmanoglu, F. (2018). Effects of organosolv pretreatment conditions for lignocellulosic biomass in biorefinery applications: a review. Journal of renewable and sustainable energy, 10, 1-22. doi:10.1063/1.5025876

Nele Verhulst, I., \& Bram Govaerts, F. (2015). Agricultura de conservación, ¿mejora la calidad del suelo a fin de obtener sistemas de producción sustentables? Centro internacional de Mejoramiento de Maíz y Trigo, CIMMYT. Recuperado de https://repository.cimmyt.org/bitstream/handle/1088 3/4408/56985.pdf?sequence $=4 \&$ is Allowed $=y$

Parejo Famés, C., Aparicio, A., \& Albaladejo, R. G. (2019). Una aproximación a la ecología epigenética en plantas. Ecosistemas. Revista cientifica de ecología y medio ambiente, 28(1), 69-74. doi:10.7818/ECOS.1605

Pérez Cruz, O. (2020). La competitividad y la seguridad alimentaria en México. Economía Coyuntural, 5(1), 119-142. Recuperado de http://www.scielo.org.bo/pdf/ec/v5n1/v5n1_a06.pdf

Pokhrel, S., Shrestha, M., Slouf, M., Sirc, J., \& Adhikari, R. (2020). Eco-friendly urea-formaldehyde composites based on corn husk cellulose fiber. International Journal of Composite Materials, 29-36. doi:10.5923/j.cmaterials.20201002.01

Prado, M. M., Azaldo , H. J., Becerra , A. B., Palacios, J. H., Vargas, R. J., \& Rentería, U. M. (2012). Caracterización de hojas de mazorca de maíz y de bagazo de caña para la elaboración de una pulpa celulósica mixta. Madera y Bosques 18 (3), 2012:37-51, 42. Recuperado de http://www.scielo.org.mx/pdf/mb/v18n3/v18n3a4.pd f

Qi, H. (2017). Novel Functional Materials Based on Cellulose. Springer. doi:DOI 10.1007/978-3-31949592-7

Rewini Kunusa, W., Isa , I., AR Laliyo, L., \& Iyabu , H. (2018). FTIR, XRD and SEM analysis of microcrystalline cellulose (MCC) fibers from corncobs in alkaline treatment. Journal of Physics: Conference Series, 1-8. doi:10.1088/17426596/1028/1/012199

Sebe, G., Ham Pichavant , F., Ibarboure , E., Chantal Koffi , A., \& Tingaut , P. (2012). Supramolecular Structure Characterization of Cellulose II Nanowhiskers Produced by Acid Hydrolysis of Cellulose I Substrates. BioMacromolecules, 570-578. doi:10.1021/bm201777j 
Sierra Macías , M., Andrés Meza , P., Rodríguez Montalvo, F. A., \& Espinosa Calderon, A. (2016). Introgresión genética de genotipos mejorados en maíces nativos de las razas Tuxpeño y Olotillo con calidad de hoja del totomoxtle. Revista de Aplicación Científica y Técnica, 2(4), 45-52. Recuperado de https://www.ecorfan.org/spain/researchjournals/Apli cacion_Cientifica_y_Tecnica/vol2num4/Revista_de Aplicacion_Cientifica_y_Tecnica_V2_N4_7.pdf

Singh , G., Jose , S., Kaur, D., \& Soun, B. (2020). Extraction and Characterization of Corn Leaf Fiber. (T. Group, Ed.) Journal of natural fibers, 1-11. doi:10.1080/15440478.2020.1787914

Singh , H. K., Patil , T., Vineeth , S. K., Das , S., Pramanik, A., \& Mhaske, S. T. (2019). Isolation of microcrystalline cellulose from corn stover with emphasis on its constituents: corn cover and corn cob. Materials Today: Proceedings. doi:10.1016/j.matpr.2019.12.065

Sosa , B. A., \& Ruíz Ibarra, G. (2017). La disponibilidad de alimentos en México: un análisis de la producción agrícola de 35 años y su proyección para 2050. SciELO, 207-230. doi:https://doi.org/10.22185/24487147.2017.93.027

Torres Jaramillo, D., Morales Vélez, S. P., \& Quintero Díaz, J. C. (2017). Evaluación de pretratamientos químicos sobre materiales lignocelulósicos. Ingeniare. Revista Chilena de ingeniería, 25(4), 733-743. Recuperado de https://scielo.conicyt.cl/pdf/ingeniare/v25n4/07183305-ingeniare-25-04-00733.pdf

United States Department of Agriculture. (2021). World Agricultural Production. Foreign Agricultural Service. Recuperado de https://apps.fas.usda.gov/psdonline/circulars/product ion.pdf
Velázquez G., J. J., Salinas G., J. R., Potter, K. N., Gallardo V., M., Caballero H., F., \& Díaz M., P. (2002). Cantidad, cobertura y descomposición de residuos de maíz sobre el suelo. TERRA Latinoamericana, 20(2), 171-182. Recuperado de https://www.redalyc.org/pdf/573/57320210.pdf

Velázquez López, L., Salgado García, S., Turrado Saucedo, J., Hidalgo Moreno, C., Ortíz García, C., Córdova Sánchez, S., Saucedo Corona A. R., Canché Escamilla, G. (2020). Celulosa y microcelulosa de residuos del cultivo de caña de azúcar (Saccharum spp.). Agro Productividad, 13(4), 11-17. doi:10.32854/agrop.vi.1591

Yepes, A., \& Silveira Buckeridge , M. (2011). Respuestas de las plantas ante los factores ambientales del cambio climático global (revisión). Colombia forestal, 14(2), 213-232. Recuperado de https://www.redalyc.org/pdf/4239/423939616005.pd $\mathrm{f}$

Yue, Y., Han , J., Han , G., Zhang, Q., French, A. D., \& Wu, Q. (2015). Characterization of cellulose I/II hybrid fibers isolated from energycane bagasse during the delignification process: Morphology, crystallinity and percentage estimation. Carbohydrate Polymers, 133, 438-447. doi:10.1016/j.carbpol.2015.07.058

Zhang, R., \& Liu, Y. (2018). High energy oxidation and organosolv solubilization for high yield isolation of cellulose nanocrystals (CNC) from Eucalyptus hardwood. Scientific Reports, 8, 1-11. doi:10.1038/s41598-018-34667-2 